\title{
Performance Evaluation of the Lionet Quality of Service in Nsukka Campus of the University of Nigeria
}

\author{
Udora N. Nwawelu*, Anthony N. Nzeako, Mamilus A. Ahaneku, Vincent C. Chijindu \\ Department of Electronic Engineering, Faculty of Engineering, University of Nigeria, Nsukka, Enugu, Nigeria \\ Email: $\underline{\text { udodegreat@yahoo.com }}$
}

Received 8 December 2013; revised 8 January 2014; accepted 8 February 2014

Copyright (C) 2014 by authors and Scientific Research Publishing Inc.

This work is licensed under the Creative Commons Attribution International License (CC BY). http://creativecommons.org/licenses/by/4.0/

cC) (i) Open Access

\begin{abstract}
Efficient performance of the Lionet in Nsukka campus of the University of Nigeria may be hindered by the ornamental trees that characterises the physical features of the University environment. This work measured and analyzed the Lionet quality of service $(Q 0 S)$ in terms of received power level and path loss as functions of distance, under free space condition and in the presence of a tree. To achieve this, Ordinary Least Squares (OLS) regression analysis was used on the measured data with the help of SPSS version 16.0 software; to show the significance of the presence of trees on the Lionet QoS. The findings revealed that path loss is influenced significantly if $(p<0.05)$ by distance $(b=0.920)$ and effect of a tree $(b=-0.185)$, while for received power level by distance $(b$ $=-0.920)$ and effect of a tree $(b=-0.185)$. This study has revealed that unless the University takes urgent steps to provide more access points, the QoS cannot be improved since the trees are also required in the environment as wind breakers.
\end{abstract}

\section{Keywords}

Lionet, QoS, Path Loss, Received Power Level, Access Point

\section{Introduction}

The University of Nigeria, Nsukka (UNN) in Enugu state of Nigeria, through the University ICT unit has embarked on extending internet access to all parts of the campus, by deploying access points (APs) at various locations within the campus. Lionet is a campus wireless local area network (WLAN) preferred by the University of Nigeria, Nsukka governing council for her staff and students. This network is preferred due to its positive as-

\footnotetext{
${ }^{*}$ Corresponding author.

How to cite this paper: Nwawelu, U.N., et al. (2014) Performance Evaluation of the Lionet Quality of Service in Nsukka Campus of the University of Nigeria. Int. J. Communications, Network and System Sciences, 7, 122-129. 
pects which includes: flexibility when compared to wired networks, low cost when compared to other wireless systems such as cellular data services, rapid deployment, backwards compatibility with older laptop and desktop computers, and familiarity to users [1]. But its efficient performance is being affected by the trees that appear within the vicinity of both transmitting and receiving antennas.

It is a known fact that when radio wave impinges on an obstruction, some of the energy can be reflected back into the initial medium, some may be transmitted into the second medium, some may be absorbed by the medium, some may be diffracted and some may be scattered [2]-[6]. The interactions of radio waves with the obstructions reduce its received signal strength (received power level). For the fact that obstruction reduces the received signal strength of a wireless network, the obstruction of trees ought to be taken into consideration when deploying wireless communication networks in the University environment.

Lionet operates on $2.4 \mathrm{GHz}$ and $5 \mathrm{GHz}$ microwave frequencies. For efficient performance of wireless network of such kind, the RF signal must reach the receiver with some minimum power (usually $-75 \mathrm{dBm}$ ) after experiencing attenuation due to: passage through air (free space loss) and passage through or refraction around the objects in its paths. Also, the AP-to-user range varies from a theoretical maximum of $3.5 \mathrm{~km}$ for cell phones, to a practically-achievable outdoor maximum of less than 200 meters [1].

The undesirable poor signal reception, loss of data packets, and delay in uploading and downloading of data, were frequently experienced in the University, which have been as a result of manmade infrastructures and meteorological phenomena. For the trees, the signal experiences absorption and scattering from the randomly distributed leaves, twigs, branches, and tree trunks [6] [7]. Figure 1 is a photograph of an AP sited close to a tree at the Nsukka campus of the University of Nigeria.

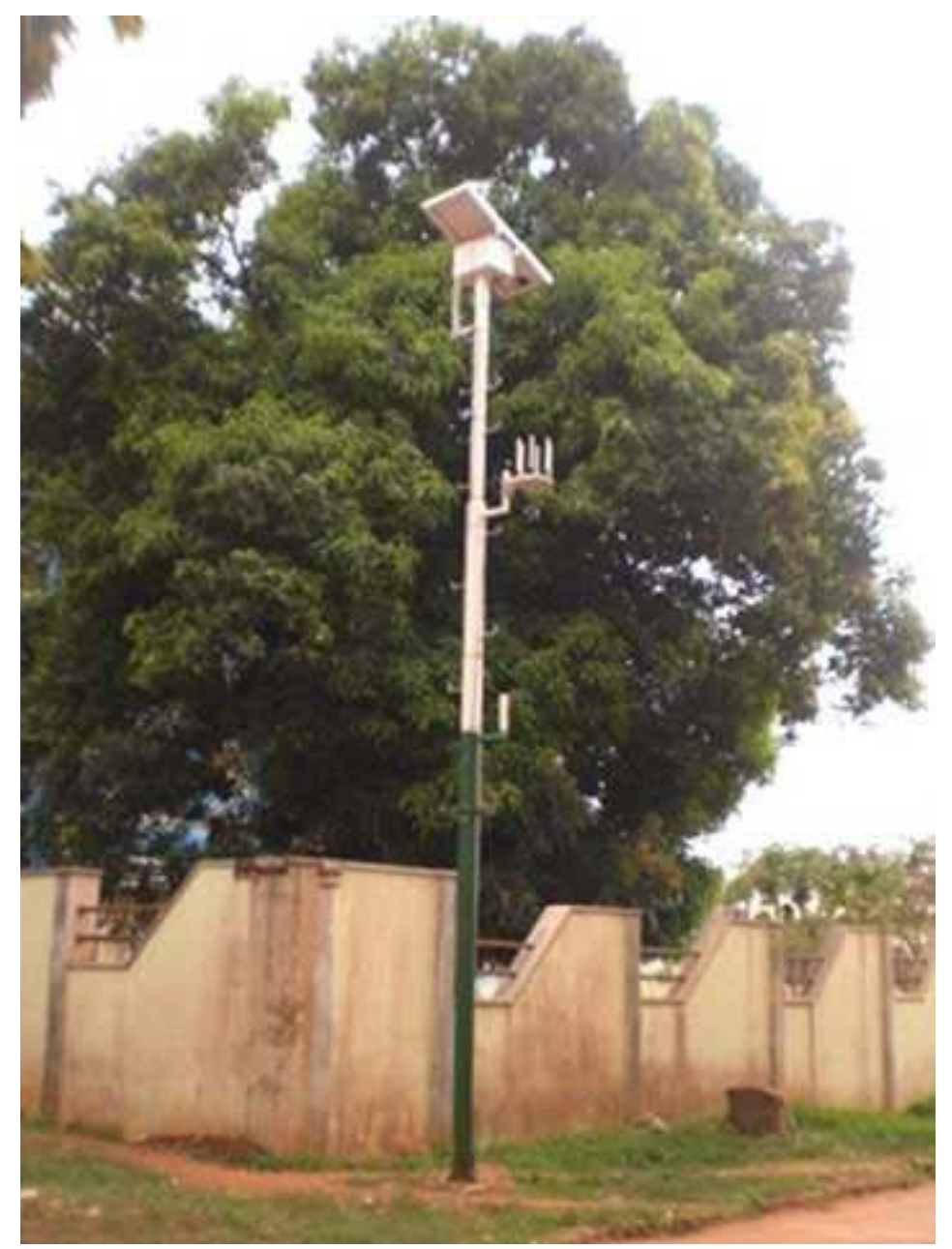

Figure 1. Access point sited close to a tree within the campus. 
We took out time to interview some of the users of Lionet on the quality of signal reception. Some of them affirmed that the signal is better around trees located near the access point (APs). This supports the notion that the QoS of Lionet is being affected by the trees which results in its scattering and absorption, thus preventing it from covering the maximum expected range. Even, there are complaints that some people who used to receive Lionet before no longer receive due to the reasons adduced above.

Numerous studies on measurement of wireless network quality have been done. The relevant literature reviewed in this paper emphasized on the effects of tree obstructions on wireless network quality [6] [8]-[11]. Investigation of foliage effects on the quality of the propagated radio signal via data logging for point-to-point link at $5.8 \mathrm{GHz}$ was reviewed. The result showed that the link which was obstructed by a single tree and a row of trees recorded an average of $12.2615 \mathrm{~dB}$ and $4.6306 \mathrm{~dB}$ excess path losses respectively [8]. In [12], study on the influence of trees on radio channel at frequencies of 3 and $5 \mathrm{GHz}$ shows that the excess path loss was between 1 and $16 \mathrm{~dB}$. Evaluation of the effects of moisture in trees was studied at frequency of $3.5 \mathrm{GHz}$, though somewhat higher than the frequency used in WLAN. It shows that a wet tree attenuated the signal considerably more (loss of $18 \mathrm{~dB}$ ) than a dry tree (loss of $11 \mathrm{~dB}$ ) [13]. Studies on the limitations of campus wireless networks at the University of Nigeria Nsukka were carried out. The result showed that an average of $6.476 \mathrm{~dB}$ excess path loss was contributed by the tree obstruction.

Among all the literatures reviewed, none has carried out statistical study on the significance of trees on radio waves being propagated. This study investigates the QoS of Lionet at University environment. The QoS was measured by the variation in received power level and path loss as functions of distance under free space condition and in the presence of a tree, using spectrum analyzer (SPECTRAN HF 6080). The measured data with the help of SPSS version 16.0 software were statistical analysed, to show the significance of the presence of trees on the Lionet QoS.

\section{Theoretical Consideration}

Quality of Service (QoS) is the measure which defines the performance in any accountable system. Depends on the particular type of application, QoS can be considered by reliability, robustness, availability, security, and among others [14]. The QoS of Lionet are measured and analysed based on their received power level and path loss all in (dBm).

\subsection{Received Power Level}

It is the power level in $(\mathrm{dBm})$ that is received at the receiver within cell site. The capability of the radio receiver to pick up the required level of radio signals will allow it to operate more efficiently within a radio communication network system. Received power level can be related to many parameters of the radio communications system namely; path loss, transmitting power, antenna gain, and many others. The expression for the received power level from the transmitting antenna to a receiving antenna is written as:

$$
P_{r}=P_{t}-P L_{F S}
$$

where,

$P_{r}=$ Power received level in $\mathrm{dBm}$

$P_{t}=$ Power transmitted in $\mathrm{dBm}$

$P L_{F S}$ : Free space path loss in $\mathrm{dBm}$

Equation (1) can only be employed under a free space condition.

\subsection{Path Loss (PL)}

Path loss is an unwanted introduction of energy tending to interfere with the proper reception and reproduction of the signals when propagated from the source to the sink. It reduces the power density of an electromagnetic wave as it propagates through space [14]-[16]. Path loss is one of the important parameters that can be employed in analysis of wireless network performance. Since this paper is investigating the contribution of trees on the QoS of Lionet, Equation (1) is extended.

The path loss of Lionet signal passing through a tree includes; summation of free space path loss and excess loss (tree loss factor) [6] [8] [17]. We considered the tree loss factor to account for the increase in attenuation of the received signal when the receiver is placed behind a tree. Mathematically, the expression becomes [6]: 


$$
P L_{T R E E}=P L_{F S}+L_{T R E E}
$$

where,

$P L_{\text {TREE }}=$ Path loss in the presence of a tree.

$L_{F S}=$ Free space loss.

$L_{\text {TREE }}=$ Tree loss factor.

Substituting Equation (2) in (1) we have

$$
P_{r}=P_{t}-P L_{F S}-L_{T R E E}
$$

Furthermore Equation (3) becomes

$$
P_{r}=P_{t}-P L_{T R E E}
$$

Free Space Path Loss (PLFS)

Free space path loss model $\left(P L_{F S}\right)$ is usually the reference model employed under an ideal condition. This loss occurs as the signal travels from transmitter to receiver through space without any other effects attenuating the signal [14] [15] [18] [19]. The value used to compute free space is dependent only on the distance that is from the transmitting antenna. In free space, the path loss increases by $20 \mathrm{~dB}$ per decade [20]. The equation for free space propagation loss between two antennas is given as [21]:

$$
\left(\frac{P_{R}}{P_{T}}\right)=\left(\frac{\lambda}{4 \pi R}\right)^{2}
$$

where,

$P_{R}=$ Power received

$P_{T}=$ Power transmitted

$R=$ Transmitting and receiving antennas distance of separation.

\section{Materials and Methods of Measurements}

A portable EMF (electromagnetic frequency) measurement system, Spectran HF 6080 model was used to intercept the signal from Lionet AP. The equipment consists of three main component parts namely: Hyper Log Measurement antenna, Spectran HF 6080 (Aaronia AG software). It is worthy of note that the Spectran HF 6080 was connected to laptop for data logging. This is because the Spectran HF 6080 has low memory. This forms the receiving part at a frequency of $2400 \mathrm{MHz}$.

The Lionet AP contains an Omni-directional antenna (WBS 2400) with transmitting power of $19 \mathrm{~dB}$ [22]. The transmitting antenna was mounted at the top of a mast. Part of the Lionet AP of interest is free from tree obstruction while part is impeded by a tree. Both scenarios are shown in Figure 2 and Figure 3 respectively. We obtained the measured path loss from the power level received.

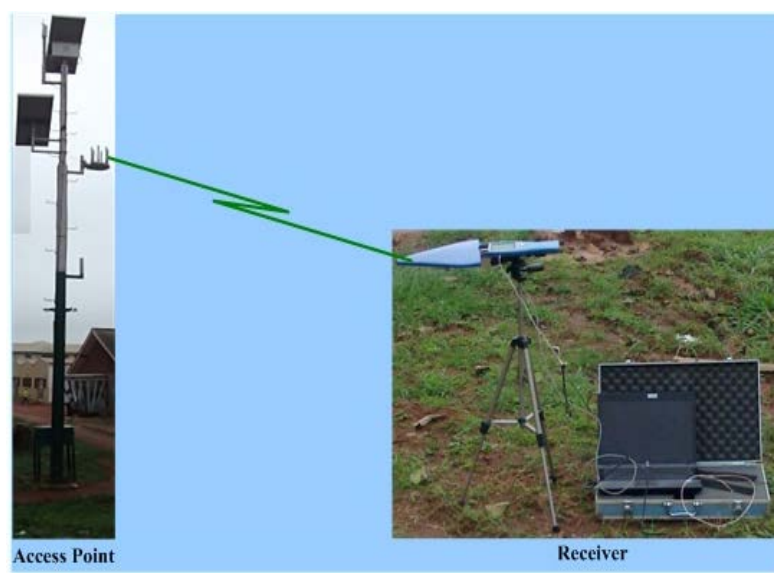

Figure 2. Free space condition.

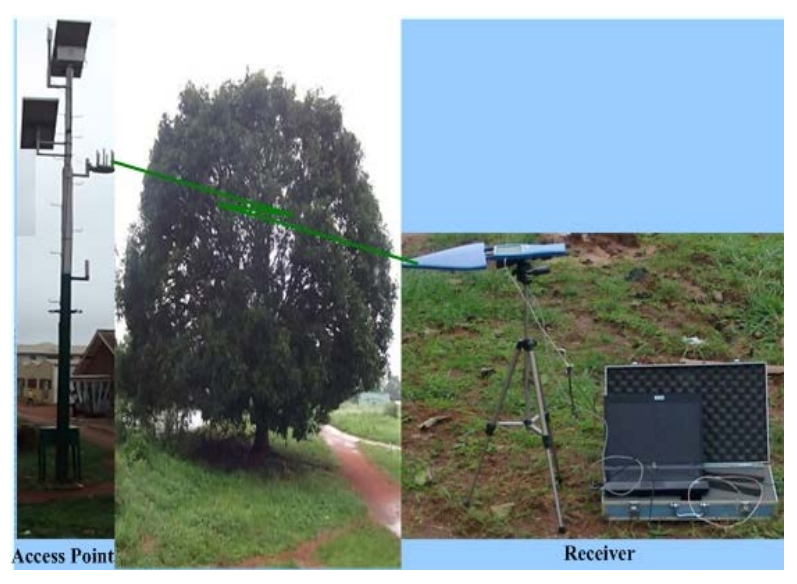

Figure 3. Obstruction of Lionet signal by a tree. 


\section{Method of Data Analysis}

The data used in the analysis of this work were collected at every 20 meters away from the Lionet AP. At every 20 meters away from the AP, 25 data points were logged. From the measured received power level, we obtained the measured path loss. The data collected for both scenarios are represented graphically in Figure 4 and Figure 5 respectively.

\section{Statistical Analysis of Results}

Ordinary Least Square (OLS) regression analysis is used on the data collected to reveal an undesirable effect of trees on Lionet Qos. Regression analysis was carried out using linear function under free space condition and multiple regressions when there is tree obstruction. The dependent variables and independent variable are expressed as follows:

$$
\begin{gathered}
P r=b_{0}+b_{1} D \\
\operatorname{Pr}=b_{0}+b_{1} D+b_{2} T \\
P L=b_{0}+b_{1} D \\
P L=b_{0}+b_{1} D+b_{2} T
\end{gathered}
$$

where received power level $(P r)$ and path loss $(P L)$ equal the dependent variables, Distance $(D)$ and the effect of a tree $(T)$ equal the independent variable, and $b$ equals the regression parameters or coefficients.

The regression parameters are computed using SPSS version 16.0.

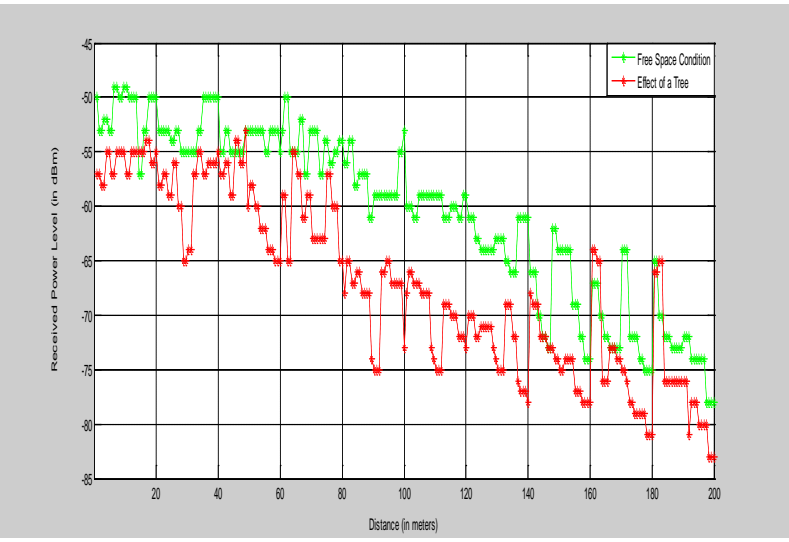

Figure 4. Variation of Lionet received power level under free space condition and in the presence of a tree.

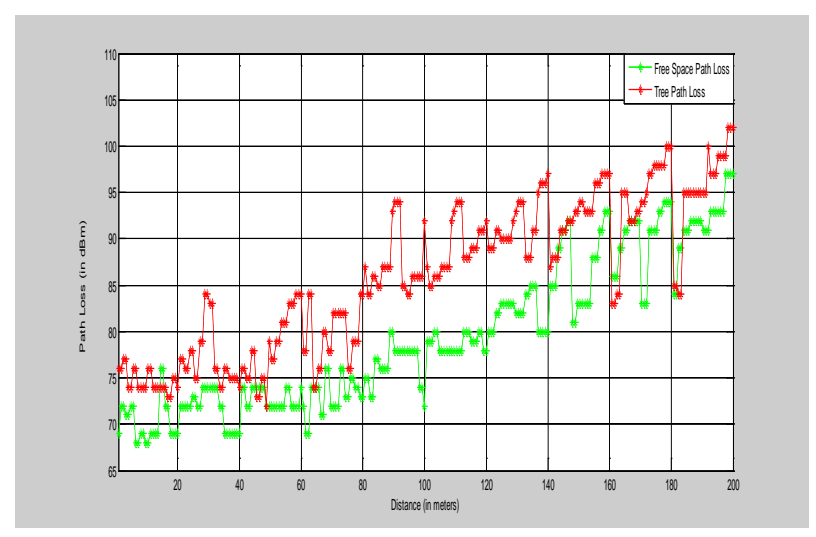

Figure 5. Variation of Lionet path loss under free space condition and in the presence of a tree. 


\section{Statistical Results and Discussions}

The data collected for this study were analyzed, summarized and presented in this section. The results were tabulated to enhance understanding and explicit interpretations. They are presented in Tables 1-4.

As shown in Table 1, the constant term has a negative sign which implies that, if other variable is assumed to be zero, the received power level under free space condition decreases at a constant rate of -46.357 . The distance under free space condition has a negative sign. It shows that decrease in distance leads to an increase in received power level. The coefficient of the distance is -0.929 showing that a unit increase in distance under free space condition will lead on the average to a $-92.9 \%$ decrease in the received power level. The $\mathrm{R}^{2}$ was 0.862. This implies that the independent variable (Distance) accounted for $86.20 \%$ of the total variation of the dependent variable (Received power level under free space condition). The remaining $13.8 \%$ is the residual and this could be due to error and other factors not investigated. The F-value $(1554.146 ; \mathrm{P}<0.05)$ is significant at $5 \%$, implying that the model is significant.

In Table 2, the constant term has a negative sign which implies that, if other variables are assumed to be zero, the received power level in the presence of a tree decreases at a constant rate of -48.672 . The distance in the present of a tree has a negative sign. It shows that decrease in distance leads to an increase in its received power level. The coefficient of the distance in the presence of a tree is -0.920 showing that a unit increase in its distance will lead on the average to a $-92.0 \%$ decrease in its received power level. The effect of a tree has a negative sign. It reveals that absent of tree leads to an increase in received power level. The coefficient of the effect of a tree is -0.185 showing that a unit increase in the number of trees will lead on the average to a $-18.5 \%$ decrease in the received power level. The $\mathrm{R}^{2}$ was 0.897 . This implies that the independent variables (Distance and Effect of a Tree) accounted for $89.70 \%$ of the total variation of the dependent variable (Received power level due to the effect of a tree). The remaining $10.3 \%$ is the residual and this could be due to error and other factors not investigated. The F-value (1071.386; $\mathrm{P}<0.05)$ is significant at $5 \%$, implying that the model is significant.

Table 3 is the summary of OLS regression results for free space path loss. As shown in the table, the constant term has a positive sign which implies that, if other variable is assumed to be zero, the path loss under free space condition increases at a constant rate of 65.357. The distance under free space condition has a positive sign. It shows that distance has a positive relationship with free space path loss. An increase in distance equally will lead to increase in free space path loss. From the table, coefficient of the distance is 0.929 showing that a unit increase in distance under free space condition will lead on the average to a $92.9 \%$ increase in the free space path loss. $\mathrm{R}^{2}$ is 0.862 . This implies that the independent variable (Distance) accounted for $86.20 \%$ of the total variation of the dependent variable (Free Space Path loss). The remaining 13.8\% is the residual and this could be due to error and other factors not investigated. The F-value (1554.146; P < 0.05) is significant at 5\%, implying that the model is significant.

In Table 4, the constant term has a positive sign which implies that, if other variables are assumed to be zero, tree path loss increases at a constant rate of 67.672. The distance in the present of a tree has a positive sign. It shows that increase in distance leads to an increase in its path loss. The coefficient of the distance in the presence of a tree is 0.920 showing that a unit increase in its distance will lead on the average to a $92.0 \%$ increase in its path loss. The effect of accounted a tree has a positive sign. It reveals that present of a tree leads to an increase in path loss. The coefficient of the effect of a tree is 0.185 showing that a unit increase in the number of trees will lead on the average to a $18.5 \%$ increase in the tree path loss. $\mathrm{R}^{2}$ is 0.897 , this implies that the independent variables (Distance and Effect of a Tree) for $89.70 \%$ of the total variation of the dependent variable (Tree path loss). The remaining $10.3 \%$ is the residual and this could be due to error and other factors not investigated. The F-value (1071.386; $\mathrm{P}<0.05)$ is significant at $5 \%$, implying that the model is significant.

\section{Conclusions}

This work has studied the effect of trees on QoS of the Lionet in terms of path loss and received power level. This was achieved through field measurments under free space condition and in the presence of a tree. The measured data were statistically analyzed using Ordinary Least Square regression and SPSS version 16.0 software. From the measurement campaign that were carried out under the two scenarios, up to 200 meters, the attenuation of the Lionet signal under free space condition did not reduce the received signal strength below -75 $\mathrm{dBm}$ which is the minimum value for a reliable WLAN connection of such type while above 180 meters; at- 
tenuation of the Lionet signal in the presence of a tree reduced the received signal strength below $-75 \mathrm{dBm}[1]$.

Furthermore, the study through the help of statistical analysis revealed that: the effect of a tree and distance predicted the variation in received power level and path loss during the period under study, which agrees with our earlier preliminary investigation. Also, the results of the simple and multiple regression analyses could therefore be very useful in predicting the effect of distance and tree on the received power level and path loss.

Based on these observations, we concluded that the presence of trees along the Lionet propagation path significantly attenuated signals thereby prevented the network from covering its maximum expected range. This

Table 1. Summary of OLS regression results for received power level under free space condition.

\begin{tabular}{|c|c|c|c|c|c|}
\hline \multicolumn{6}{|c|}{$\begin{array}{l}\text { Dependent Variable: Received Power Level under Free Space Condition } \\
\text { Predictors: (Constant), Distance } \\
\text { Date: 15-Dec-2012, Time: 10:18:33; Observations: } 250\end{array}$} \\
\hline Variable & Coefficients & Std. Error & t-statistic & Sig. & Decision \\
\hline Constant & -46.357 & 0.405 & -114.510 & 0.000 & Significant \\
\hline Distance & -0.929 & 0.003 & -39.423 & 0.000 & Significant \\
\hline
\end{tabular}

Source: Computed from Collected data. $\mathrm{F}=1554.146 . \mathrm{R}=0.929$; $\mathrm{R}$ Square $=0.862$; Adjusted $\mathrm{R}$ Square $=0.862$. Standard Error of the Estimate $=$ 2.963. ${ }^{*}$ Significant at 5 percent.

Table 2. Summary of OLS regression result for received power level in the presence of a tree.

\begin{tabular}{cccccc}
\hline \multicolumn{5}{c}{$\begin{array}{c}\text { Dependent Variable: Received Power Level Due to the Effect of a Tree. } \\
\text { Predictors: (Constant), Distance, Effect of a Tree } \\
\text { Date: 15-Dec-2012, Time: 10:26:50; Observations: } 250\end{array}$} & Decision \\
\hline Variable & Coefficients & Std. Error & t-statistic & Sig. & Significant \\
& -48.672 & 0.435 & -111.945 & 0.000 & Significant \\
Constant & -0.920 & 0.003 & -44.903 & 0.000 & Significant \\
Distance & -0.185 & 0.037 & -9.048 & 0.000 & \\
Effect of a Tree & & &
\end{tabular}

Source: Computed from Collected data. $\mathrm{F}=1071.386 . \mathrm{R}=0.947$; $\mathrm{R}$ Square $=0.897$; Adjusted R Square $=0.896$. Standard Error of the Estimate $=$ 2.57311. * Significant at 5 percent.

Table 3. Summary of OLS regression results for free space path loss.

\begin{tabular}{cccccc}
\hline \multicolumn{5}{c}{$\begin{array}{c}\text { Dependent Variable: Free Space Path loss. } \\
\text { Predictors: (Constant), Distance } \\
\text { Date: 15-Dec-2012, Time: 10:45:00; Observations: } 250\end{array}$} \\
\hline Variable & Coefficients & Std. Error & t-statistic & Sig. & Decision \\
\hline Constant & 65.357 & 0.405 & 161.443 & 0.000 & Significant \\
Distance & 0.929 & 0.003 & 39.423 & 0.000 & Significant \\
\hline
\end{tabular}

Source: Computed from Collected data. $\mathrm{F}=1554.146 . \mathrm{R}=0.929$; $\mathrm{R}$ Square $=0.862$; Adjusted $\mathrm{R}$ Square $=0.862$. Standard Error of the Estimate $=$ 2.96307. * Significant at 5 percent.

Table 4. Summary of OLS regression results for tree path loss.

\begin{tabular}{cccccc}
\hline \multicolumn{5}{c}{$\begin{array}{c}\text { Dependent Variable: Tree Path loss. } \\
\text { Predictors: (Constant), Distance, Effect of a Tree } \\
\text { Date: 15-Dec-2012, Time: 10:50:13; Observations: } 250\end{array}$} \\
\hline Variable & Coefficients & Std. Error & t-statistic & Sig. & Decision \\
\hline Constant & 67.672 & 0.435 & 155.644 & 0.000 & Significant \\
Distance & 0.920 & 0.003 & 44.903 & 0.000 & Significant \\
Effect of a Tree & -0.185 & 0.037 & -9.048 & 0.000 & Significant \\
\hline
\end{tabular}

Source: Computed from Collected data. $\mathrm{F}=1071.386 . \mathrm{R}=0.947$; $\mathrm{R}$ Square $=0.897$; Adjusted $\mathrm{R}$ Square $=0.896$. Standard Error of the Estimate $=$ 2.57311. * Significant at 5 percent. 
study therefore recommends that the effects of trees should be taken into consideration while deploying the Lionet APs. This can be achieved by increasing the density of APs to compensate for the presence of trees in the environment.

\section{References}

[1] Dobkin, D.M. (2005) RF Engineering for Wireless Networks: Hardware, Antennas, and Propagation. Newness, San Francisco, 448.

[2] Rappaport, T.S. (2000) Wireless Communication, Principles and Practice. 2nd Edition, Pearson Education Inc., Singapore.

[3] Meng, Y.S., Lee, Y.H. and Ng., B.C. (2009) Study of Propagation Loss Prediction in Forest Environment. Progress in Electromagnetics Research B, 17, 117-133. http://dx.doi.org/10.2528/PIERB09071901

[4] Abebe, T. (2010) Modeling of Scattering and Absorption by Phantom Tissue. Addis Ababa University Addis Ababa, Ethiopia.

[5] Hie, S.S. (2004) Radio Channel Modeling for Mobile Ad Hoc Wireless Networks. Naval Postgraduate School Monterey, California.

[6] Nwawelu, U.N., Nzeako, A.N. and Ahaneku, M.A. (2012) The Limitations of Campus Wireless Networks: A Case Study of University of Nigeria, Nsukka [Lionet]. International Journal of Networks and Communications, 2, 112-122. http://dx.doi.org/10.5923/j.ijnc.20120205.04

[7] Meng, Y.S., Lee, Y.H. and Ng, B.C. (2009) Study of Propagation Loss Prediction in Forest Environment. Progress in Electromagnetics Research B, 17, 117-133. http://dx.doi.org/10.2528/PIERB09071901

[8] Ly, P.L., Ly, P.L., Rahman, T.A. and Abu, M.K. (2010) Investigation of Foliage Effects via Remote Data Logging at 5.8 GHz. WSEAS Transactions on Communication, 9, 237-247.

[9] Chukwudi, O.R. (2008) The Effects of Vegetation on GSM Signal Propagation in Rural Areas. Master Thesis Dissertation, University of Nigeria Nsukka, Nigeria.

[10] Gans, M.J., Amitay, N., Yeh, Y.S., Damen, T.C., Valenzuela, R.A., Cheon, C. and Lee, J. (2002) Measurements for Fixed Wireless Loops (FWL) in a Suburban Region with Foliage and Terrain Blockages. IEEE Transactions on Wireless Communications, 1, 302-310. http://dx.doi.org/10.1109/7693.994824

[11] Perras, S. and Bouchard, L. (2002) Bouchard: Fading Characteristics of RF Signals Due to Foliage in Frequency Bands from 2 to $60 \mathrm{GHz}$. The 5th International Symposium on Wireless Personal Multimedia Communications, 27-30 October 2002, 267-271

[12] Karlsson, A., Schuh, R.E., Bergljung, C., Karlsson, P. and Lowendahl, N. (2001) The Influence of Trees on Radio Channel at Frequencies of 3 and 5 GHz. IEEE VTS 54th Vehicular Technology Conference, Atlantic City, 7-11 October 2001, 2008-2012.

[13] Lacan, I. and McBride, J.R. (2009) City Trees and Municipal Wi-Fi Networks: Compatibility or Conflict?” Scientific Journal of the International Society of Arboriculture, Arboriculture \& Urban Forestry, 35, 203-210.

[14] Sharma, D. and Singh, R.K. (2012) Analysis of Handover Initiation using Path Loss to Sustain QoS. International Journal of Scientific \& Engineering Research, 3, 1-6.

[15] Sharma, D. and Singh, R.K. (2010) The Effect of Path Loss on QoS at NPL. International Journal of Engineering Science and Technology, 2, 3018-3023

[16] Sharma, P.K. and Singh, R.K. (2012) Analysis of Large Scale Propagation Models \& RF Coverage Estimation. International Journal of Computer Networks and Wireless Communications (IJCNWC), 2, 204-208.

[17] Saunders, S.R. and Aragon-Zavala, A. (2007) Antennas and Propagation for Wireless Communication Systems. John Willey \& Sons, Ltd., London.

[18] Sharma, D. and Singh, R.K. (2011) A Modified Way of Evaluating Loss to Sustain the QoS in Highly Populated Area. International Journal of Advanced Science and Technology, 29, 9-16.

[19] Sharma, D., Sharma, P.K. and Singh, R.K. (2012) Modified Approach to Estimate the Propagation Path Loss in Urban Area. International Journal of Advances in Electronics Engineering, 2, 275-279.

[20] Smith, C. (2000) Wireless Telecom FAQs. McGraw-Hill, New York.

[21] ITU-R (1994) Recommendation ITU-R. Conversion of Annual Statistics to Worst-Month Statistics. International Telecommunication Union, Geneva, 841.

[22] http://wavionnetworks.com/innerdata/pdf/Introduction DT antenna.pdf 